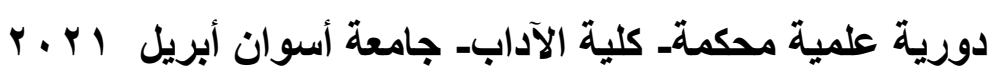

\title{
Problèmes de traduction des proverbes
}

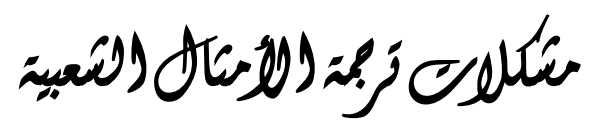

$$
\begin{aligned}
& \text { 9)(?) }
\end{aligned}
$$

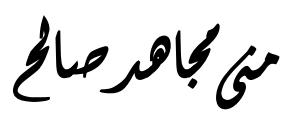

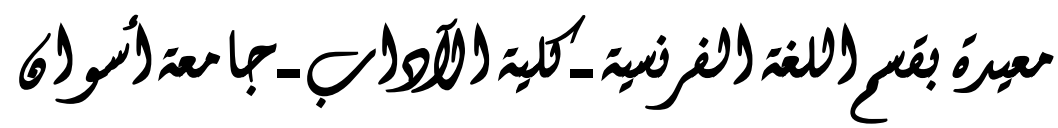

$$
\begin{aligned}
& \text { البروبرالإكترونة: } \\
& \text { monamegahed94@yahoo.com }
\end{aligned}
$$




\section{Résumé de la recherche}

La traduction littérale des proverbes se heurte à quelques problèmes. Elle change quelquefois le message du proverbe parce que le sens des mots se modifie selon le contexte. Dans cet article, nous essayons donc de traiter les problèmes de traduction où nous expliquons les messages des proverbes français et leurs équivalents arabes. En outre, nous proposons une traduction nouvelle pour les proverbes du corpus. Nous essayons de découvrir les équivalents des proverbes français au sein de la société arabe et vice-versa. Le lecteur français, par l'emploi du proverbe arabe, essaie de découvrir les sentiments et le langage de l'homme égyptien, de même, le locuteur arabe, par l'emploi du proverbe français, peut aussi connaitre la culture française. Pour cela, nous montrerons ici l'équivalent arabo-égyptien des quelques proverbes français par les deux langues française et arabe qui peuvent exprimer les mêmes réalités par différentes expressions. On étudiera les proverbes selon deux traductions: la prononciation (translittération) et le sens. 


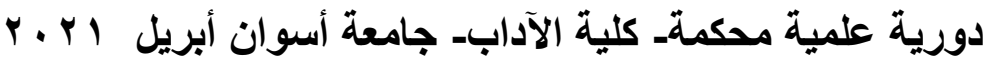

\section{ملخص البحث}

لا شئ أجمل تأثير آ من الحكم والأمثال المنتقاه من أعذب الكلام. الأمثال الشعبية هي إحدى نواتج التزاث الشعبى علي مر العصور. و لا زلنا نستدل بمثل شعبى عبارة عن جملة من كلمات قليلة على موقف كبير نحكيه في أيام كثيرة. إذاً الأمثال الشعبية من الأشياء التي نستخدمها يومياً وتسهل علينا أساليب التعبير فى حياتتا اليومية وتتقلنا إلى مستوى أعلى من التفاهم. قد تسبب الترجمة الحرفية للأمثال الشعبية بعض مشكلات الترجمة. حيث إنها يمكن أن تغير سياق المثل وذلك لأن معنى الكلمات يتغير حسب كل سياق. نحاول فى هذا

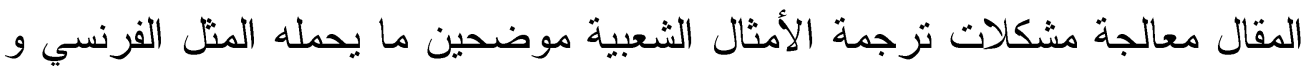
مكافئه العربى. مستخدمين الترجمة الدالية باحثين عن المكافئ العربي لبعض

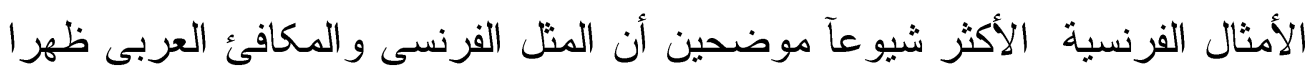
فى فتر ات مختلفة بأشكال مختلفة لفظياً ونحوياً معبرين عن نفس النصيحة. يحاول القارئ الفرنسي ، من خلال استخدام المثل العربي ، اكتشاف مشاعر ولغة الرجل المصري ، وبالمثل يمكن للمتحدث باللغة العربية ، من خلال استخدام المثل الفرنسي ، معرفة الثقافة الفرنسية. من أجل ذلك ، سنعرض هنا المعادل العربي المصري لبعض الأمثال الفرنسية باللغتين الفرنسية والعربية الاتى تعبر ا عن نفس الحقائق بتعابير مختلفة. سوف ندرس الأمثال حسب ترجمتين: النطق (الترجمة الصوتية)

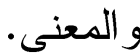




\section{Problèmes de traduction des proverbes :}

Lorsque nous traduisons les proverbes, nous affrontons naturellement quelques problèmes relatifs au sens parce que le traducteur doit donner une traduction fidèle, montrant le but du proverbe :

«Comme le traducteur de poésie, le traducteur de proverbes affronte un problème ardu : Il doit donner une traduction fidèle et qui sonne comme un proverbe $\gg{ }^{(1)}$.

Pour donner la traduction, il faut connaitre au moins deux cultures : une pour la langue source (langue arabe) et l'autre pour la langue cible (langue française). D'autre part, on ne peut pas traduire littéralement les proverbes parce que ce type de la traduction se heurte aux obstacles (il faut traduire son rythme, sa longueur, son allitération), ou il change peut -être le message du proverbe (il donne la même traduction mais il ne donne pas sa morale), c'est-à-dire qu'on peut traduire tous les mots du texte mais cette traduction ne donne probablement aucun sens. Néanmoins on peut trouver l'idée du texte dont les mots ne sont pas traduits (littéralement) parce que la valeur sémantique, la syntaxe et les grammaires ne sont pas les mêmes dans toutes les langues :

«Nous avons rencontré
des problèmes lexicaux
parce que, d'une part, le
mot français n'a pas
toujours un équivalent
précis en arabe; et
d'autre part, le mot
intégré dans une
expression doit être

1 - PRIVAT (Maryse), A propos de la traduction des proverbes, In Revista de Fitología Romaníca, número 15 , janvier 1998, p286. 


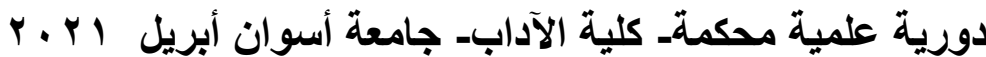

traduit selon les mots qui

l'entourent ${ }^{(1)}$.

Alors, le sens des mots se modifie selon le contexte, par conséquent, le phénomène de la polysémie apparaitra. Voici un exemple :

-Ahmed achètera une nouvelle voiture.

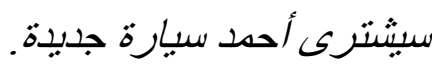

-Ce journaliste a annoncé une fausse nouvelle.

أ علن هذا الصحفى خبراً كاذبًا.

On constate immédiatement que le mot «nouvelle» est traduit dans la première phrase par (2) "جديدة", adjectif qui signifie «inconnue » ${ }^{(3)}$, c'est-à-dire : «apparaitre la chose pour la première fois ${ }^{(4)}$, mais dans la seconde phrase on le traduit par (5) "خبر qui signifie «information» : "premier avis qu'on donne ou qu'on reçoit d'un évènement récent, cet évènement porté pour la première fois à la connaissance du public » ${ }^{(6)}$. Alors, avant la traduction du mot, il nécessite de comprendre le contexte où il est situé :

«De ce fait, le premier pas

de la démarche traductrice

sera non pas un travail

linguistique sur les mots

1- SAAD ALI (Mohamed), En attendant Godot de Samuel Beckett, traduction en arabe et étude littéraire et linguistique, thèse de magistère, Faculté des Lettres, Université de Zagazig, Egypte,2008, p 215.

ـ عبد النور (جبور)، إدريس (سهيل)، قاموس المنهل فرنسي عربي، الطبعة الثامنة، دار الأداب،

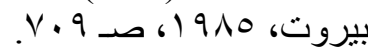

- ABDELNOR (Jabor), EDRIS (Sohail) dictionnaire français-arabe, $8^{\text {ème }}$ version, Dar ALAdab, Beyrouth, 1985, P709.

3 - BERTAUD DU CHAZAUD (Henri), dictionnaire de synonymes et contraires, les usuels, paris, 2001, P493.

4 -C. F MORVAN (Danièle), REY (Alain), «Dictionnaire LE ROBERT», France loisirs, paris, 1994. P774.

-D'après le dictionnaire HACHETTE: Nouvelle: «qui n'existais pas auparavant, avant telle date donnée ».

$$
\text { • - عبد النور (جبور )، إدريس (سهيل)، مرجع سابق، ص } 9 \text { • V. }
$$

- ABDELNOR (Jabor), EDRIS (Sohail), OP.CIT. P709.

6 - MORVAN (Danièle), REY (Alain), OP.CIT. P774. 


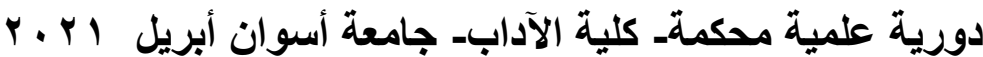

mais une recherche
bibliographique, á savoir
rechercher l'équivalent (ou
les équivalents) préexistant
dans la langue d'arrivée.
Lorsque cet équivalent
existe, il faudra aussi tenir
compte du degré
d'utilisation différent d'une
langue àl'autre "(1)

En effet, les cultures nous guident à traduire correctement l'équivalent de l'autre langue. Si l'on comprend bien le contexte du proverbe, on trouvera facilement son équivalent. En plus, il y a deux sens du proverbe: un sens explicite (figuré) que le destinataire comprend facilement et un autre implicite (propre) que l'on comprend conformément au contexte :

«Dans le processus de la traduction des proverbes, la première étape sera, non pas une analyse des mots ou des images, mais la recherche, en premier lieu, du sens exact du proverbe, hors contexte et dans son contexte ${ }^{(2)}$

C'est pour cette raison qu'on préfère parler d'équivalences proverbiales plutôt que de traduction. Dans ce chapitre, on s'interroge sur le sens figuré et propre du proverbe Mais si l'on ne trouve pas d'équivalent pour un proverbe, il permet généralement d'utiliser la traduction sémantique :

1 - PRIVAT (Maryse), OP.CIT. P283.

2 -MOHAMAD El SAYED El TOUKHY (Dalia), L'interculturalité entre le proverbe populaire égyptien et français, In Magazine de Faculté des lettres de linguistiques et de cultures comparées, V12, N² 2, Egypte, 2020, P944. 
«Face aux proverbes

n'ayant pas d'équivalent

attesté, nous avons signalé

que la solution $d u$

dictionnaire se limitant à

une traduction sémantique

était souvent plate sur le

plan stylistique; c'est alors

que le traducteur peut se

montrer créatif et écrire un

proverbe à l'aide de la

langue d'arrivée » ${ }^{(1)}$.

En un mot, par les proverbes, nous pouvons comprendre les sentiments et la culture de l'autre, ses habitudes, son niveau économique, son niveau social et son folklore où le proverbe donne un plus grand réalisme :

"Les littératures de toutes les

époques se sont servies de ces

formes sentencieuses dans des buts

bien différents : trait de folklore, critique d'une idée populaire, caractérisation du langage d'un personnage, etc. Mais il y a eu aussi des proverbes qui ont été l'inspiration des auteurs, étant donné que leur caractère pédagogique et moralisateur leur convenait dans des situations littéraires bien déterminées » ${ }^{(2)}$.

Donc, le proverbe est nettement un lieu de rencontre de la langue et de la culture. Pour cela, nous pensons que la connaissance du proverbe représente l'expérience de l'individu :

1 - QUITOUT (Michel) \& MUNOZ (Julia Sevilla), Traductologie, proverbes et figements, Le Harmattan, paris, 2009, p46.

2 - CONDE TARRÍO (Germán), La littérature et le proverbe, le proverbe et la littérature, Universidade de Santiago de Compostela, In Littérature, langages et arts: rencontres et création, 2017, p10. 


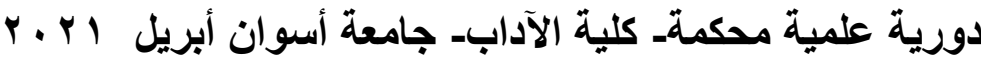

"Chaque personne enrichit son fonds de proverbes en même temps qu'elle multiplie ses expériences de vie. Les proverbes nous ont toujours impressionnés par leurs formes simples mais ils peuvent faire écho à une grande diversité de situations, de jugements. Nous nous sommes aussi étonnée qu'une telle exactitude dans l'image s'accompagne d'une telle souplesse dans l'interprétation. Il s'agit là d'une parole commune mais qu'il faut savoir prononcer au moment favorable. Nous avons voulu comprendre d'où vient cette force énigmatique ${ }^{(1)}$.

C'est pourquoi, dans le présent chapitre, nous essayons de découvrir les équivalents des proverbes français au sein de la société arabe et vice-versa. Le lecteur français, par l'emploi du proverbe arabe, essaie de découvrir les sentiments et le langage de l'homme égyptien, de même, le locuteur arabe, par l'emploi du proverbe français, peut aussi connaitre la culture française. Pour cela, nous montrerons ici l'équivalent arabo-égyptien des quelques proverbes français par les deux langues française et arabe qui peuvent exprimer les mêmes réalités par différentes expressions. On étudiera les proverbes selon deux traductions: la prononciation (translittération) et le sens.

Passons à l'analyse de ce proverbe :

1 - THI HUONG (Nguyen), «De la production du sens dans le proverbe, analyse linguistique contrastive d'un corpus de proverbes contenant des praxemes corporels en français et en vietnamien ", Thèse de doctorat, Université Paul-Valery, France, 2008, p17. 


\begin{tabular}{|c|c|c|}
\hline $\begin{array}{c}\text { «Il ne faut pas } \\
\text { allonger la jambe } \\
\text { plus loin que ne peut } \\
\text { aller la couverture } \\
\end{array}$ & "على قلّ لحافٔك مد رد & $\begin{array}{c}\text { clâ qad liḥ̂âak mid } \\
\text { riĝ́lak }\end{array}$ \\
\hline \multicolumn{3}{|c|}{ Autres formes du même contexte } \\
\hline $\begin{array}{l}\text { 1-« Selon le vent, la } \\
\text { voile } »^{3)} \text {. } \\
2-« \text { Selon le bras fait } \\
\text { la saignée» }{ }^{(4)} \text {. } \\
\text { 3-« Gouverne ta } \\
\text { bouche selon ta } \\
\text { bourse }{ }^{(5)}\end{array}$ & 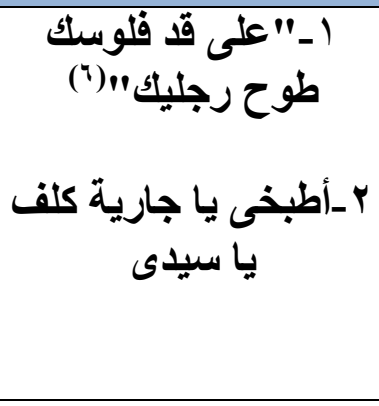 & $\begin{array}{l}\text { 1-'lâ qad filusaq } \\
\text { tawh riĝlyk } \\
\text { 2-'țbuhy yâ } \\
\text { gâryah kalif yâ } \\
\text { sydy }\end{array}$ \\
\hline
\end{tabular}

Le sens explicite montre qu'il ne doit pas étendre nos corps hors de la couverture. Par le sens implicite, ce proverbe illustre qu'il faut être satisfait de sa vie et savoir comment la diriger, soit on a beaucoup d'argent, soit on a peu d'argent. Il ne faut donc pas acheter des choses qui ne nous profitent pas, Le même message apparait clairement dans l'équivalent. Le sens du proverbe est le

1 - ABDEL HADI (Mohammed), «Recueil de proverbes français et leurs équivalents en arabe et en anglais », Egypte, IMP. Salah El Dine Alex 1933. $\mathrm{P} 76, \mathrm{n} 400$

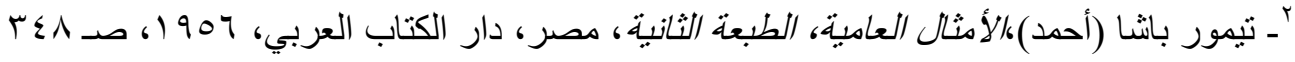

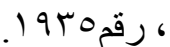
-TAYMOR BACHA (Ahmed), proverbs populaires, 2 $2^{\text {ème }}$, Egypte, 1956, P348, N¹935.

3 - ALATTAR (Abdallah), les proverbes populaires français, Egypte, Atlas de l'édition et la production médiatique, 2013. P56.

4 - MOHAMAD El SAYED El TOUKHY (Dalia), OP.CIT. P956.

5 - INMACUADA (María) Enseigner et apprendre les unités parémiologiques d'une langue étrangère : du XIXe siècle à nos jours, In Cédille, $\mathrm{N}^{\circ} 10$, Université de Rovirai Virgili, Espagne 2014, P 339.

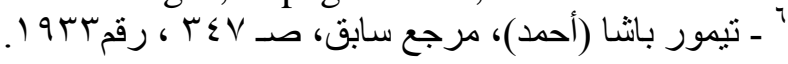

- TAYMOR BACHA (Ahmed), OP.CIT. P347, N 1933. 
même mais l'emploi des mots et des expressions s'énumèrent. Dans le cas précédent, le verbe «allonger» signifie «étendre un membre " ${ }^{(1)}$ qui exprime le mouvement. Dans le cas suivant, il désigne aussi (prolonger : faire durer plus longtemps) qui exprime le temps :

L'équivalent arabe de ce proverbe peut être :

$$
\text { «Le pas court allonge la vie }{ }^{(2}
$$

"حرة صبرت فى بيتها عمرت "( " (")

(Hura şabarit fy bythâ ${ }^{c}$ marit)

En outre, le verbe «allonger» peut exprimer la continuité de la vie. Alors, le message de ce proverbe (sens implicite) semble pertinent : la patience aide l'homme à réussir.

Un autre sens métaphorique s'adresse à celui qui sait attendre, qui peut obtenir finalement tout ce qu'il veut et il réalise par conséquent ses buts :

\begin{tabular}{|c|c|c|}
\hline $\begin{array}{c}\text { «Les petits } \\
\text { ruisseaux font les } \\
\text { grandes rivières» }{ }^{(4)}\end{array}$ & رحلة الألف ميل تبدأ & $\begin{array}{l}\text { Rehlt el'lf myl } \\
\text { tbd' by hatwa. }\end{array}$ \\
\hline \multicolumn{3}{|c|}{ Autres formes du même contexte } \\
\hline
\end{tabular}

Le sens explicite du proverbe cité ci-dessous montre que quelqu'un parle mais en vain :

"Il est des sots pour lesquels il

n'existe aucun espoir de salut. Il

est inutile de perdre son temps en

cherchant à les bonifier. Ce

1 - MORVAN (Danièle), REY (Alain), OP.CIT. P30.

2 - http://www.cultivonsnous.fr/100385-le-pas-court-allonge-la-vie. Consulté le 5-5-2020.

-TAYMOR BACHA (Ahmed), OP.CIT. P195, Nº1049.

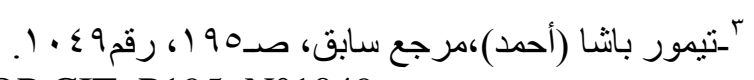

4 - ALATTAR (Abdallah), OP.CIT. P41.

5 - NILLA (Sowmiya) Les proverbes à travers le monde, ÉLODIL, paris 2006, P.15.

${ }^{6}$ - AL ATTAR (Abdallah), OP.CIT. P76. 
دورية علمية محكمة كلية الآداب- جامعة أسوان أبريل ا.r.

proverbe s'utilise pour exprimer

son dépit face à un caractère, qui

malgré nos efforts, ne change pas

ou face à un entêtement

inconsidéré » ${ }^{(1)}$ :

\begin{tabular}{|c|c|c|}
\hline $\begin{array}{c}\text { Prêcher dans le } \\
\left.\text { désert » }{ }^{2}\right)\end{array}$ & - بيأذن في مالطا. & $\begin{array}{l}\text { bi’zin fy } \\
\text { mâlța }\end{array}$ \\
\hline \multicolumn{3}{|c|}{ Autres formes du même contexte } \\
\hline $\begin{array}{l}\text { A laver la tête d'un âne } \\
\text { l'on y perd que sa lessive }\end{array}$ & علم في المتبلم يصبح & $\begin{array}{c}\text { clim fy } \\
\text { âlmitbalim } \\
\text { yşbaḥ nâsy }\end{array}$ \\
\hline
\end{tabular}

Notre société arabe s'enrichit des proverbes qui portent le même contexte du proverbe précédent :

$$
\text { ينفخ في قربة مقطو عة }
$$

(Yunfuh fy qirbah maqț ${ }^{c} a h$ )

D’après le dictionnaire Robert : le verbe «prêcher » signifie « recommander ${ }^{(3)}$, ou «conseiller : enseigner la révélation religieuse ${ }^{(4)}$. Le même verbe traite une autre question dans le cas suivant :

\begin{tabular}{|c|c|c|}
\hline $\begin{array}{c}\text { «Si on jugeait les gens à } \\
\text { la barbe, le bouc pourrait } \\
\text { prêcher » }\end{array}$ & "اللي تقول عليه & $\begin{array}{l}\text { 'lly tiqul clyh } \\
\text { musâ yṭlac } \text { Firc }^{c} \text { un }\end{array}$ \\
\hline \multicolumn{3}{|c|}{ Autres formes du même texte } \\
\hline$\ll L$ & 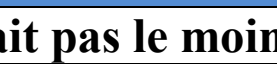 & \\
\hline
\end{tabular}

Le verbe «Prêcher» signifie aussi "prôner : essayer de convaincre, faire la morale à quelqu'un » ${ }^{(8)}$. Il a pour but de

1 - http://www.linternaute.fr/proverbe/385/a-laver-la-tete-d-un-ane-1-on-yperd-que. Consulté le 23-5-2021.

${ }^{2}$ - ABDEL HADI (Mohammed), OP.CIT. P143, n 741.

3 - BERTAUD DU CHAZAUD (Henri), OP.CIT. P558.

4 - MORVAN (Danièle), REY (Alain), OP.CIT. P886.

5 - http://www.linternaute.fr/proverbe/recherche/jugement. Consulté le 9-92020.

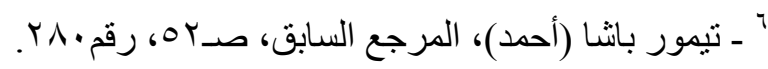

-TAYMOR BACHA (Ahmed), OP.CIT. P52. N²80.

7- ABDEL HADI (Mohammed), OP.CIT. P 117, n 618.

8 - MORVAN (Danièle), REY (Alain), OP.CIT. P886. 
montrer que l'idée de l'apparence physique peut influencer notre jugement d'une personne. Cette idée est faussée, il ne faut pas juger l'homme selon l'apparence.

Outre, le verbe «prêcher » peut exprimer la défense :

\begin{tabular}{|c|c|c|}
\hline $\begin{array}{c}\text { Chacun prêche de } \\
\text { sa paroisse » }\end{array}$ & أنا ومن بعدى الطوفان & $\begin{array}{c}\text { 'nâ wi min bedy } \\
\text { âl-țufân }\end{array}$ \\
\hline \multicolumn{3}{|c|}{ Autres formes du même cotexte } \\
\hline $\begin{array}{c}\text { Chacun porte sa } \\
\text { croix en ce monde }^{(2)}\end{array}$ & 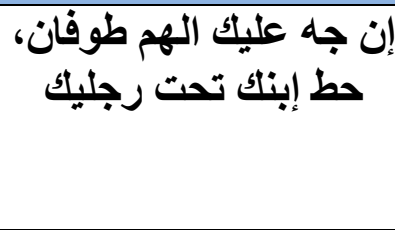 & $\begin{array}{c}\text { 'in ĝah 'lyk âl- } \\
\text { ham țufân ḥuṭ } \\
\text { 'bnak taḥt } \\
\text { riĝ̀lyk }\end{array}$ \\
\hline
\end{tabular}

Ce proverbe montre que l'homme fait tout ce qu'il est possible pour contenter lui-même ou exactement chacun s'occupe de résoudre ses problèmes personnels. D'autre part, face aux problèmes, on tente de répondre à cette question : qu'est- ce qu'on peut faire ? on pense, on cherche mais on ne peut rien faire. Tout à coup, une solution très simple donnée, c'est $A L L A H$ qui nous dispose le moyen. Le message du proverbe suivant montre qu'il faut avoir une confiance que notre seigneur ne nous laisse jamais : «Dieu porte son secours aux gens qui sont dans le désespoir » ${ }^{(3)}$ :

\begin{tabular}{|c|c|c|}
\hline $\begin{array}{c}\text { «A barque } \\
\text { désespérée, Dieu } \\
\text { fait trouver le } \\
\text { port » }\end{array}$ & والربن في أدم في التفكير & $\begin{array}{l}\text { 'bn 'dam fy âltafkyr we } \\
\text { âl-rab fy âltadbyr. }\end{array}$ \\
\hline \multicolumn{3}{|c|}{ Autres formes du même contexte } \\
\hline $\begin{array}{l}\text { «' L'homme } \\
\text { propose et Dieu } \\
\text { dispose » }\end{array}$ & 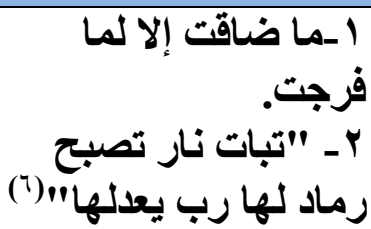 & $\begin{array}{c}\text { 1-mâ d̦âqat 'lâ lamâ } \\
\text { furiôat } \\
\text { 2- Tibât nâr tişbah } \\
\text { ramâd liha rab y'dlhâ }\end{array}$ \\
\hline
\end{tabular}

1- MOY (Sylvie), 100 Proverbes français, Franc-Parler, Sénas, France, 2012. P27.

2 -ABDEL HADI (Mohammed), OP.CIT. P 33, n 179.

3 - http://www.linternaute.fr/proverbe/3991/a-barque-desesperee-dieu-faittrouver-le-port. Consulté le 29-5-2021.

4 -ABDEL HADI (Mohammed), OP.CIT. P1, n 1 .

5 __QUITARD (P.M), OP.CIT. P311.

$$
\text { " - تيمور باثا (أحمد)،مرجع سابق، صـ 109 ، رقم 119 }
$$




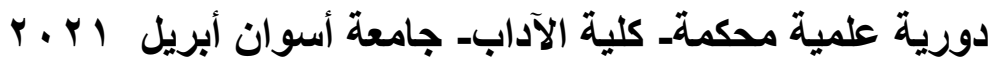

Le mot «désespérée » signifie «perdue » ${ }^{(1)}$ la barque (qui signale un petit bateau) n'a plus d'espoir ${ }^{(2)}$ du retour. D'ailleurs, le mot «barque » occupe dans les proverbes une place prépondérante :

\begin{tabular}{|c|c|c|}
\hline $\begin{array}{l}\text { « Malheur à la barque } \\
\text { que mènent de } \\
\text { capitaines » }^{(3)}\end{array}$ & المركبن اللى فيها & $\begin{array}{c}\text { âl-markib illy } \\
\text { fyha raysyn } \\
\text { tiğraq. }\end{array}$ \\
\hline \multicolumn{3}{|c|}{ Autres formes du même contexte } \\
\hline $\begin{array}{l}\text { « Autant des têtes, } \\
\text { autant d'avis » }\end{array}$ & خيطين متخيطش" "الإبرة اللى فيا" & $\begin{array}{l}\text { âl'b } \\
\text { hytt. }\end{array}$ \\
\hline
\end{tabular}

Le sens explicite du proverbe cité ci-dessus montre l'idée que : Deux capitaines à bord d'un navire le font toujours sombrer. C'est un proverbe à sens métaphorique qui indique implicitement que la diversité des chefs, c'est-à-dire des opinions entravent le travail.

D'autre part, ALLAH ne nous donne jamais ce que nous ne pouvons pas supporter. "Allah n'impose à aucune âme une charge supérieure à sa capacité » ${ }^{(6)}$. Il nous donne toujours la patience pour porter les difficultés jusqu'à la fin où toute chose sera bien. C'est le message du proverbe cité ci-dessous :

\begin{tabular}{|c|c|c|}
\hline $\begin{array}{c}\text { «A brebis tondue, } \\
\text { Dieu mesure le vent }\end{array}$ & ـ"ربك رب العطا بيدي البرد الغطا") & $\begin{array}{l}\text { Rabak rab âl- } \\
\text { cțâ bidy âl- } \\
\text { bard câl qad } \\
\text { âlógatâ }\end{array}$ \\
\hline
\end{tabular}

-TAYMOR BACHA (Ahmed), OP.CIT. P159. N869.

1 - BERTAUD DU CHAZAUD (Henri), OP.CIT. P527.

2 - MORVAN (Danièle), REY (Alain), OP.CIT. P832.

3 - http://www.linternaute.fr/proverbe/5396/malheur-a-la-barque-quemenent-beaucoup-de-capitaines. Consulté le 30-8-2019.

${ }^{4}$ - AL ATTAR (Abdallah), OP.CIT. P 67.

- TAYMOR BACHA (Ahmed), OP.CIT. P3. ํำ 16

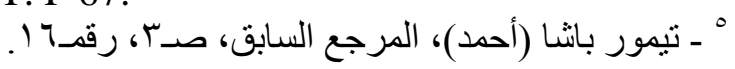

6 - https://coran12-21.org/fr/sourates/s2. Le Coran, traduction «Hamidullah révisée ,2000». Sourate «La vache » verste 286. Consulté le_30-9-2020.

7. QUITARD (P.M), OP.CIT. P 175. On dit aussi : A brebis tondue, Dieu ménage le vent.

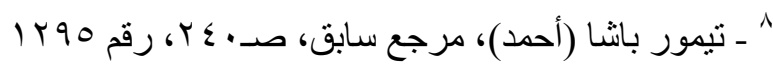

- TAYMOR BACHA (Ahmed), OP.CIT. P240. N 1295. 
Les verbes «mesurer, ménager» expriment le soin et la force d'ALLAH. Ils montrent que la divinité peut faire abattre le malheur. En ce qui concerne le mot «Brebis», il signifie "agnelle, vacive ${ }^{(1)}$ :

\begin{tabular}{|c|c|c|}
\hline $\begin{array}{c}\text { « La mort des } \\
\text { loups, c'est la santé } \\
\text { des brebis }{ }^{(3)}\end{array}$ & 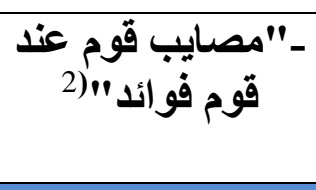 & $\begin{array}{c}\text { Maşâyb qaum cnd } \\
\text { qaum fawâ'd }\end{array}$ \\
\hline \multicolumn{3}{|c|}{ Autres formes du même contexte } \\
\hline $\begin{array}{l}\text { 1- « A quelque chose } \\
\text { malheur, est bon » } \\
\text { 2- « Le malheur des } \\
\text { uns fait le bonheur } \\
\text { des autres » }\end{array}$ & رُب ضارةُ نافُعة & rub dâratun nafcah \\
\hline
\end{tabular}

Le mot «mort» exprime le malheur du loup (les autorités injustes). Le mot «santé » exprime évidemment la liberté de l'adversaire. Le message devient clair: Le bonheur d'une personne peut causer la tristesse d'une autre : «Il y a toujours quelque avantage à retirer de nos malheurs si douloureux soient-il ${ }^{(6)}$.

Les proverbes personnifient toujours tous les détails de la vie. Dans ce même chemin, nous pouvons dire que la bonté est souvent une cause de quelques problèmes. Il est quelquefois dangereux d'avoir de douceur; les malveillants profitent de l'excessive bonté d'une personne pour l'écraser. C'est le message du proverbe suivant :

1 - BERTAUD DU CHAZAUD (Henri), OP.CIT. P135.

2 - AL ATTAR (Abdallah), OP.CIT. P86.

3 - http://www.cultivonsnous.fr/104698-la-mort-du-loup-cest-la-sante-des.

Consulté le 25-5-2021.

4 - ABDELHADI (Mohamed), OP.CIT. P10. N57.

5 - MOY (Sylvie), OP.CIT.P38.

${ }^{6}$ - EL ATTAR (Abdallah), OP.CIT. P13. 


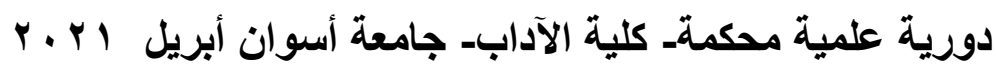

\begin{tabular}{|c|c|c|}
\hline $\begin{array}{l}\text { «Qui se fait brebis, le } \\
\text { loup le mange » }\end{array}$ & 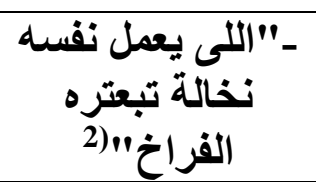 & $\begin{array}{l}\text { 'lly } y^{c} \text { mil nafsuh } \\
\text { nahâalah tibctruh } \\
\text { âlfyrâh. }\end{array}$ \\
\hline \multicolumn{3}{|c|}{ Autres formes du même contexte } \\
\hline $\begin{array}{l}\text { « Faites-vous miel, et } \\
\text { les mouches vous } \\
\text { mangeront }{ }^{(3)} \text {. }\end{array}$ & 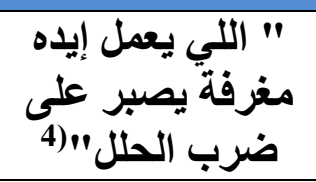 & $\begin{array}{c}\text { 'lly ycmel ’yduh } \\
\text { maǵgrafah yșbur 'lâ } \\
\text { darb âl-ḥilal }\end{array}$ \\
\hline
\end{tabular}

Selon le contexte, le mots «brebis », "miel» et les mots arabes «nahâlah, magrafah», représentent les personnages naïfs : «Trop de bonté, de générosité de votre part inciteront les autres à abuser de vous » ${ }^{(5)}$.

Passons à une autre occurrence :

\begin{tabular}{|c|c|c|}
\hline Tel père tel fils. & من شابه أباه فما ظلم. & Man šâbah 'bâh famâ zalam. \\
\hline \multicolumn{3}{|c|}{ Autres formes du même contexte } \\
\hline $\begin{array}{l}1-\text { «De doux arbre, } \\
\text { douces pommes » }(6) \\
2-\text { «Bon sang ne peut } \\
\text { mentir }(7) \\
\text { 3- « Les chiens ne font } \\
\text { pas des chats }(8)\end{array}$ & 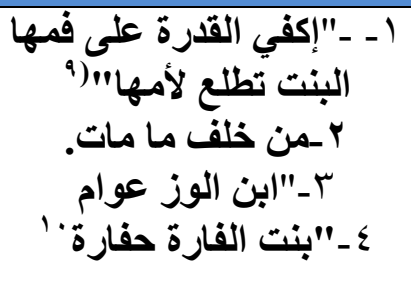 & $\begin{array}{l}\text { 1- 'kfy âl-qidrah cla fmahâ } \\
\text { âl-bent tițlac l'umahâ. } \\
\text { 2- Man halaf mâ mât. } \\
\text { 3-'bn âl-wiz c'wâm } \\
\text { 4-Bint âl-fârah ḥafârah. }\end{array}$ \\
\hline
\end{tabular}

1-ABDELHADI (Mohamed), OP.CIT. P153. N787.

- TAYMOR BACHA (Ahmed), OP.CIT. P86. N489.

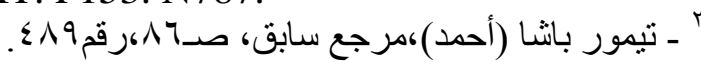

3 - CHACOTO (LUCÍlIA MARÍA), Figement et variation dans les proverbes portugais, In Paremia, V6, Madrid, 1997, P188.

$$
\text { ؛ - تيمور باشا (أحمد)، مرجع سابق، صد }
$$

- TAYMOR BACHA (Ahmed), OP.CIT. P86. N489.

5 - KODELJA (Katarina), Les expressions figées culinaires en français et leur traduction en slovène, thèse de doctorat, Université de Ljubljana, Faculté de philosophie, Ljubljana, 2019, P20.

6 -_ABDEL HADI (Mohammed), OP.CIT. P 41, n 227.

7 -QUITARD (P.M), OP.CIT. P64.

8 - MOY (Sylvie), OP.CIT. P11.

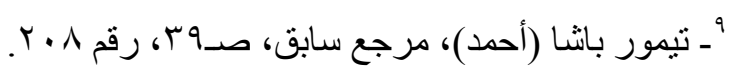

- TAYMOR BACHA (Ahmed), OP.CIT. P39. N²08.

-AMINABDELSAMD (Mohammed), OP.CIT. P474.

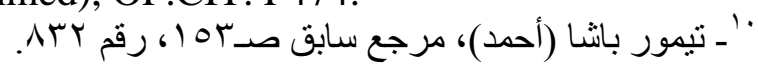

- TAYMOR BACHA (Ahmed), OP.CIT. P153. N832. 


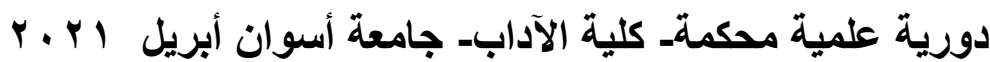

Le mot «tel » est utilisé pour exprimer la ressemblance entre les fils et les parents. Le proverbe susmentionné illustre l'idée que le fils essaie toujours d'imiter ses parents :

«On hérite des qualités et des défauts

de ses parents. Ce proverbe est surtout

utilisé pour souligner l'héritage d'un

défaut et critiquer ainsi parents et

enfants ${ }^{(1)}$.

En général, le terme «chien » est énormément utilisé dans les proverbes parce qu'il a toujours un rôle très important (Lafontaine traite plusieurs questions par le chien dans ses fables $\left.{ }^{(2)}\right)$.

Le proverbe suivant montre qu'on ne peut pas réfléchir quand on avait faim, Ou bien on rêve au moment où on a besoin :

\begin{tabular}{|c|c|c|}
\hline $\begin{array}{c}\text { « Le chien ne rêve que } \\
\text { d'os » }\end{array}$ & الجعان يحلم بسوق العيش & $\begin{array}{l}\text { âl-ĝịân } \\
\text { yḥlam bi- } \\
\text { suq âl-cyšs }\end{array}$ \\
\hline \multicolumn{3}{|c|}{ Autres formes du même contexte } \\
\hline
\end{tabular}

Il est donc évident que le proverbe égyptien appartient au registre du langage courant, alors que le français au registre soutenu.

Examinons un autre exemple :

\begin{tabular}{|l|c|c|}
\hline Gens de même farine & $\begin{array}{c}\text { mếgunyn min ținah } \\
\text { wâhdah }\end{array}$ \\
\hline
\end{tabular}

1 - http://www.linternaute.fr/proverbe/637/les-chiens-ne-font-pas-des-chats. Consulté le 29-5-2021.

2 - D'après fables «le loup et le chien », Le raisonnement du chien semble fort bon à La Fontaine : mais venant d'un simple chien : "On trouva qu'il ne valait rien » La Fontaine attribue ici au chien la faculté d'évaluer son intérêt à agir et le dépeint comme un être qui raisonne lorsqu'il choisit de ne pas défendre le poulailler. C.F. https://books.openedition.org/psorbonne/17529. Consulté le (13-8-2021).

D'après fables «Les deux Chiens et l'Ane mort », nous trouvions cette citation : Les vertus devraient être sœurs Ainsi que les vices sont frères.

3 -CENDRIER (Patrick), Des chiens et des hommes, In Publibook, France,2003, P197.

4 - AL ATTAR (Abdallah), OP.CIT. p106. 


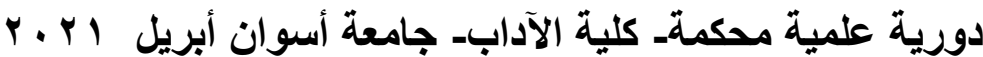

D'après le dictionnaire Robert, le terme «farine» signifie «fécule " (1), une poudre obtenue par la mouture de grains de céréales, utilisée dans la cuisine. Alors que, selon le contexte susmentionné, ce terme signifie «matière ou vase » pour montrer la similitude entre deux personnages. Ce même mot s'énumère dans les proverbes français

\begin{tabular}{|c|c|c|}
\hline $\begin{array}{l}\text { «Tout ce qui est blanc } \\
\text { n'est point farine » }\end{array}$ & "الحريز بقى سن سيد البس & $\begin{array}{c}\text { miš kul min libs } \\
\text { âl-ḥaryr biqâ } \\
\text { sayd }\end{array}$ \\
\hline \multicolumn{3}{|c|}{ Autres formes du même contexte } \\
\hline $\begin{array}{l}\text { « Tout ce qui brille n'est } \\
\text { pas or » }\end{array}$ & "مـا كل براق & $\begin{array}{c}\text { mâ kul mâ ylme } \\
\text { dhbân . }\end{array}$ \\
\hline
\end{tabular}

Ici le mot «farine » symbolise la couleur blanche. Le mot «or» symbolise la chose brillante. Dans la culture arabe, la soie a une grande valeur parce qu'elle est une matière très chère. Les deux, l'or chez les français et la soie chez les arabes, représentent implicitement la même idée : les apparences ne reflètent pas la réalité, en outre, elles sont souvent trompeuses. L'utilisation de mot « Farine » S'énumère dans les proverbes :

\begin{tabular}{|c|c|c|}
\hline$\frac{\text { «Par nuit semble tout }}{\underline{\text { blé farine }{ }^{(6)}}}$ & الليل أخفى بالويل & Al-lyl 'ḩfâ bil-wyl \\
\hline \multicolumn{3}{|c|}{ Autres formes du même contexte } \\
\hline $\begin{array}{l}\text { « La nuit, tous les chats } \\
\text { sont gris » }\end{array}$ & الليل ستار العيوب & $\begin{array}{l}\text { Al-lyl satâr âl- } \\
\text { cyub }\end{array}$ \\
\hline
\end{tabular}

1 - BERTAUD DU CHAZAUD (Henri), OP.CIT. P321.

2 http://www.linternaute.fr/proverbe/5051/tout-ce-qui-est-blanc-n-est-pointfarine. Consulté le 15-2-2020.

$$
\text { r }
$$

- AMINABDELSAMD (Mohammed), OP.CIT. P540.

${ }^{4}$-MOY (Sylvie), OP.CIT. P12. Elle existait auparavant sous la forme "tout ce qui reluit n'est pas or », puis le verbe reluire se transforma en briller pour suivre l'apanage des ménagères.

5 -AL ATTAR (Abdallah), OP.CIT. P59.

${ }^{6}$ - $\quad$ http://www.linternaute.fr/proverbe/3881/par-nuit-semble-tout-ble-farine. Consulté le 12-12-2019.

7 - QUITARD (P.M), OP.CIT. P214. 


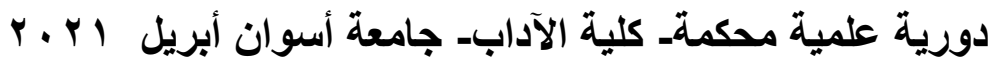

En ce qui concerne le sens explicite, on trouve que les chats ont, en nuit, la même couleur (noire ou grise). De même, on voit toutes les grains d'une couleur blanche. Alors, c'est un phénomène physiologique que tous les objets se ressemblent dans l'obscurité :

«Il n'y a point de différence pour

la vue, pendant l'obscurité, entre

les belles et les laides " ${ }^{(1)}$.

Le cas suivant retourne à l'antiquité. Les personnes s'attirent à leurs pareils parce qu'ils pensent de la même façon:" Les individus dotés de qualités et défauts semblables souvent s'associent ou forment des couples ${ }^{(2)}$ :

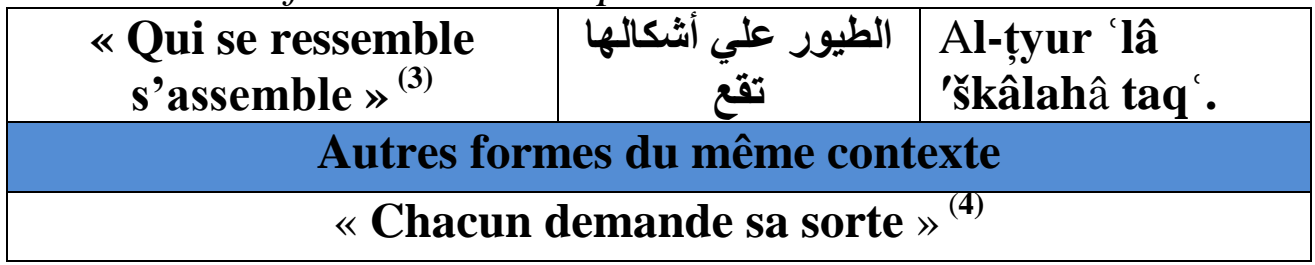

Chaque homme est conscient de ce qui se passe dans sa propre vie. Il connait bien son ami et son ennemi. C'est le message du proverbe :

\begin{tabular}{|c|c|c|}
\hline $\begin{array}{l}\text { «Chacun sait bien } \\
\text { où son soulier le } \\
\text { blesse }{ }^{(5)}\end{array}$ & 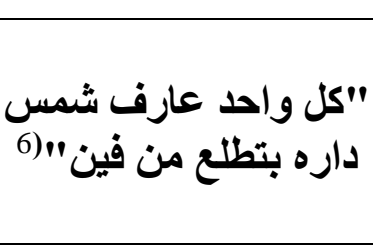 & $\begin{array}{l}\text { Kul wâḥid 'ârif } \\
\text { šams dâruh } \\
\text { btițla' min fyn }\end{array}$ \\
\hline \multicolumn{3}{|c|}{ Autres formes du même contexte } \\
\hline $\begin{array}{c}\text { "Chacun sait ses } \\
\text { affaires » } 7 \text { ) }\end{array}$ & أهل مكة أدرى بشعابها & $\begin{array}{c}\text { 'hl makah 'drâ } \\
\text { biši' âbhâ }\end{array}$ \\
\hline
\end{tabular}

1 - QUITARD (P.M), OP.CIT. P214.

2 - AL ATTAR (Abdallah), OP.CIT. P51.

3 -ABDEL HADI (Mohammed), OP.CIT. P153, n 791

- AMINABDELSAMD (Mohammed. OP.CIT. P518.

4 -ABDEL HADI (Mohammed) ${ }_{2}$ OP.CIT. P32, n 174.

5 - Ibid. P433, N 181.

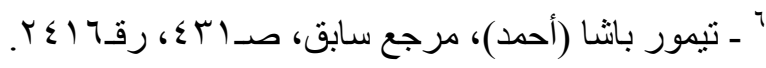

- TAYMOR BACHA (Ahmed), OP.CIT. P431. N²416.

7 - AL ATTAR (Abdallah), OP.CIT. P72. 


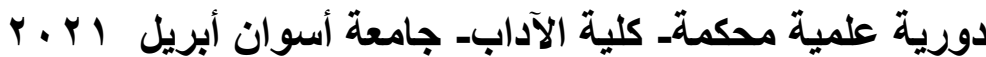

Un bon conseil apparait dans le cas suivant : chacun a le droit au respect de la vie privée :

\begin{tabular}{|c|c|c|}
\hline $\begin{array}{l}\text { «Entre le bois et } \\
\text { l'écorce, il ne faut } \\
\text { pas mettre le } \\
\text { doigt » }\end{array}$ & من تدخع ما في لايرضا لا يعنيه & $\begin{array}{c}\text { Man tadahal fymâ } \\
\text { lâ yu nyh, sam' mâ } \\
\text { lâ yurḍyh. }\end{array}$ \\
\hline \multicolumn{3}{|c|}{ Autres formes du même contexte } \\
\hline $\begin{array}{c}\text { «Qui entre au } \\
\text { moulin, en sort } \\
\text { poudré » }{ }^{(2)} \text {. }\end{array}$ & 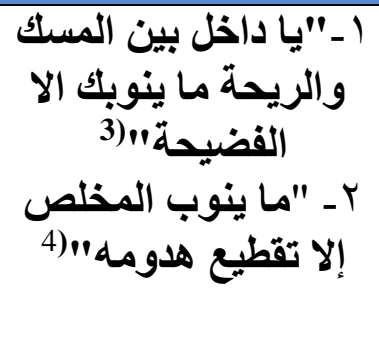 & $\begin{array}{c}\text { 1- Y dâhuel byn } \\
\text { elmesk w elryḥa mâ } \\
\text { ynwbak 'lâ elfḍyeḥa } \\
\text { 2- Mâ yenoub } \\
\text { elmah̆leş elâ taqtyc } \\
\text { hedomo }\end{array}$ \\
\hline
\end{tabular}

Molière récite le même sens, où il dit :

«Sganarelle: Apprenez que Cicéron dit, qu'entre l'arbre et le doigt, il ne faut point mettre l'écorce ${ }^{5)}$

Selon le dictionnaire Larousse, le mot «écorce» cité ci-haut signifie : "Enveloppe de certains fruits, fragment de cette enveloppe ${ }^{(6)}$. Il est fréquemment utilisé dans les proverbes : «On presse l'orange et on en jette l'écorce » ${ }^{(7)}$

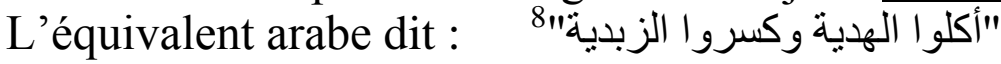

('klû âl-hidyah wi kasarû âl-zbdyah)

1 - ABDEL HADI (Mohammed), OP.CIT. P 53, N 281.

2 -Ibid, P150, N 771

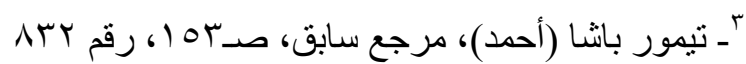

- TAYMOR BACHA (Ahmed), OP.CIT. P1531. N832.

- Ibid. P1531. N832.

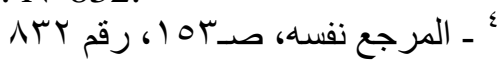

5 -_Molière, le médecin malgré lui, théâtre du palais -Royal, paris 1666, Acte 1, Sc2, P4.

6 -REY-DEBOVE (Josette), REY (Alain), dictionnaire alphabétique et analogique de la langue française, LE ROBERT, France 1993, P815.

7 - HERMAN (Jan), L'écrivain au 18 e siècle : Voltaire dans son époque, In : in Ecrivains Modes d'emploi ; De Voltaire à bleu Orange, Musée royal de Mariemont, Sofiane Laghouati, Morlanwelz, 2012, P2.

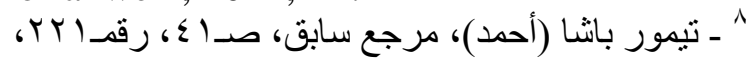

- TAYMOR BACHA (Ahmed), OP.CIT. P41. N²21. 
Le proverbe s'adresse à celui qui profite toujours les choses utiles : «'on exploite le meilleur de l'individu et après on se sépare de lui " ${ }^{(l)}$. Nous avons déjà souligné que la connaissance du proverbe est une source de l'expérience:«Chaque personne enrichit son fonds de proverbes en même temps qu'elle multiplie ses expériences de vie. ${ }^{2}$.

Alors, le mot «expérience» signifie une connaissance acquise des situations passées, une connaissance acquise par la pratique ${ }^{(3)}$. Donc, il faut prendre le conseil de l'homme expérimenté (souvent les âgés car ils sont généralement plus sages). C'est ce que se dit en français :

\begin{tabular}{|c|c|c|}
\hline $\begin{array}{l}\text { Expérience passe } \\
\text { science » }{ }^{4)}\end{array}$ & "إسأل مجرب") وهلا تسأل & $\begin{array}{l}\text { 's'l miĝarb wa lâ } \\
\text { tis'l țabyb }\end{array}$ \\
\hline \multicolumn{3}{|c|}{ Autres formes du même contexte } \\
\hline $\begin{array}{l}\text { La sagesse est une } \\
\text { mère de sciences }\end{array}$ & 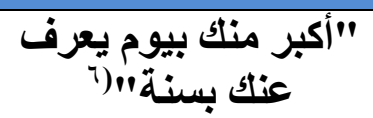 & $\begin{array}{l}\text { 'kbar minak bi-yum } \\
\text { y'raf 'anak bi-sanal }\end{array}$ \\
\hline
\end{tabular}

Les mots 《science, et طبيب représentent les conseils scientifiques. Nous voyons que la sagesse vient avec le temps "vivre, on apprend toujours quelque chose ${ }^{(7}$, c'est pourquoi, il faut d'abord consulter le médecin qui nous donne des conseils scientifiques, puis ceux qui vivent les mêmes situations. D'autre part, l'homme doit toujours être prudent. Il faut apprendre des erreurs du passé : "Quand on a déjà été victime d'un phénomène on devient plus prudent. Voire trop défiant face à un danger $d u$ même type ${ }^{(8)}$. C'est ce que se dit à travers le proverbe suivant :

1 - http://www.linternaute.fr/proverbe/3446/on-presse-1-orange-et-on-jette-1ecorce. Consulté le_7-12-2019.

2 - THI-HUONG (Nguyen), OP.CIT. P17.

3 - C.F. REY-DEBOVE (Josette), REY (Alain), OP.CIT. P1981.

4 -_ABDEL HADI (Mohammed), OP.CIT. P55, N 297.

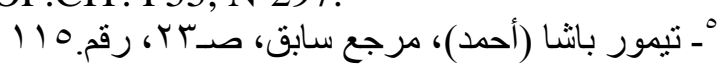

- TAYMOR BACHA (Ahmed), OP.CIT. P123. N¹15

- TAYMOR BACHA (Ahmed), OP.CIT. P37. N²03.

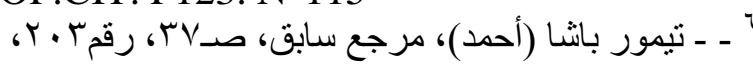

7 - AL ATTAR (Abdallah), OP.CIT. P154.

8 - Loc.cit. 


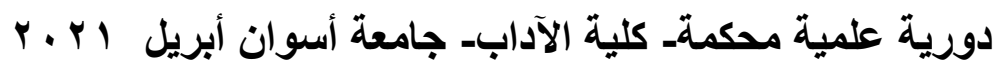

\begin{tabular}{|c|c|c|}
\hline $\begin{array}{l}\text { «Chien échaudé ne } \\
\text { revient plus en } \\
\text { cuisine } »(1)\end{array}$ & 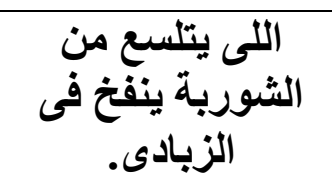 & $\begin{array}{c}\text { 'lly ytlisc min âl- } \\
\text { šurbah yunfuh fi âl- } \\
\text { zabâdy. }\end{array}$ \\
\hline \multicolumn{3}{|c|}{ Autres formes du même contexte } \\
\hline $\begin{array}{l}\text { «Chat échaudé } \\
\text { craint l'eau } \\
\text { froide }{ }^{(2)} .\end{array}$ & 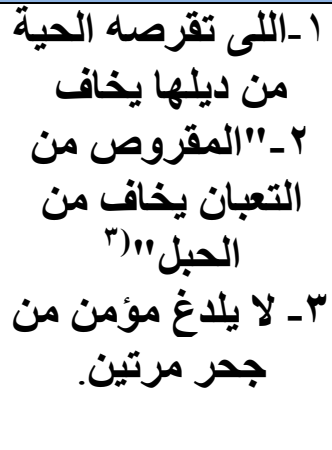 & $\begin{array}{c}\text { 1-'lly tqruşuh âl-ḥyh } \\
\text { min dylhâ yhâf. } \\
\text { 2-'Imaqruş min âl- } \\
\text { tcbân yhâf men âl- } \\
\text { ḥabl } \\
\text { 3- Lầ yuldag } \\
\text { mu' min min ĝuhr } \\
\text { martyn. }\end{array}$ \\
\hline
\end{tabular}

«Le chat échaudé » représente la personne qui a subi un mal, «l'eau froide »: c'est quelque chose d'inoffensif voire bénéfique, pour montrer que toute expérience malheureuse donne une leçon de prudence. Il est donc remarquable que le terme «chat» est énormément utilisé dans les proverbes. Dans chacun de ces proverbes, un nouveau message apparait :

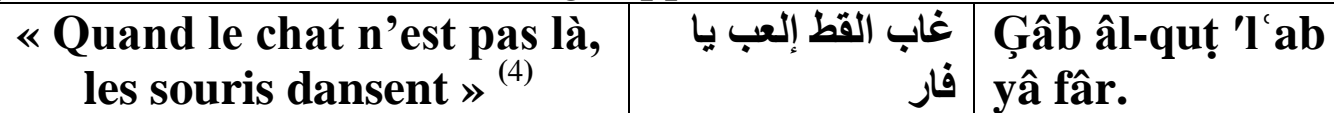

\section{Autres formes du même contexte}

\section{1- «Quand le chat est hors de la maison, les souris dansent sous la table ${ }^{(5)}$.

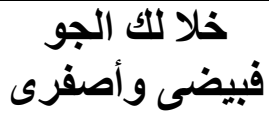 \\ hlâ lik âl-ĝaw \\ fabyḍ̂y wa 'ṣfary}

${ }^{1}$ - RENE KLEIN (Jean), La phraséologie (et en particulier les proverbes) dans le Trésor de la langue française informatisé, Paris, Nancy, conférence présentée le 24 mai 2006. P5.

2- ABDELHADI (Mohamed), OP.CIT. P35. N 192.

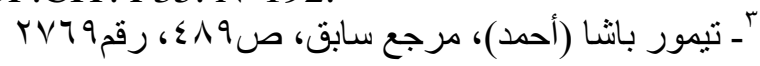

- TAYMOR BACHA (Ahmed), OP.CIT. P489. N²769.

4 - SEVILLA MUÑOZ (Julia), Les proverbes et les phrases proverbiales françaises et leurs équivalents en espagnol, In : Langages, $34^{e}$ année, ${ }^{\circ} 139$, $2000, \mathrm{P} 99$.

5 - https://www.expressions-francaises.fr/expressions-1/3355-le-chat-parti-lessouris-dansent. Consulté le 30-1-2020. 


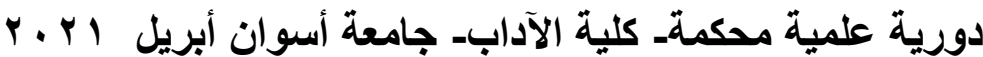

$\underline{B A L Z A C}$ cite le même sens dans son roman « Engénie Grandet » où il dit :

«Quand le chat court sur les toits; les souris dansent sur les planchers $\gg(1)$

Les verbes «danser et étaler» expriment la liberté des rats qui chantent et dansent pendant l'absence des chats. Le proverbe insiste sur l'idée que les subordonnés se reposent, lorsque le chef est absent. Les exemples sont ici nombreux : le directeur et ses employés, les parents et ses fils. Les messages du chat s'énumèrent dans les proverbes. Cet animal peut aussi symboliser la personne borgne :

$$
\text { «Appeler un chat un chat }{ }^{(2}
$$

Ce proverbe veut dire que l'homme franche n'a pas peur d'appeler les choses par leur nom. En arabe, on trouve l'équivalent qui dit:

$$
\text { (yqul lil'cwr ,'cwr fy 'cynuh). }
$$

\begin{tabular}{|c|c|c|}
\hline Proverbe français & Equivalent arabe & Translittération \\
\hline $\begin{array}{c}\text { «Qui veut noyer } \\
\text { son chien l'accuse } \\
\text { de la rage }{ }^{(4)}\end{array}$ & "لما تقع البقرة تكتر سكاكينها"(") & $\begin{array}{c}\text { Lamâ tuqc âl- } \\
\text { baqrah tiktar } \\
\text { sakâkynhâ }\end{array}$ \\
\hline
\end{tabular}

En réalité, nous inventons des torts à ceux qu'on veut débusquer.

Ce message est pertinent dans d'autres proverbes :

Le chien et la vache symbolisent le pouvoir. En un mot, l'être faible peut faire tout ce qu'il veut, quand son ennemi commence à s'affaiblir. Ce proverbe insiste aussi sur la même idée :

1 - BALZAC (Honoré de), Eugénie Grandet, roman, Alexandre Houssiaux, Éditeur, paris, 1855, P140.

2 - ABDEL HADI (Mohammed), OP.CIT. P 9, N 50.

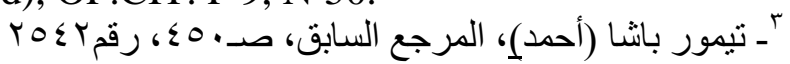

- TAYMOR BACHA (Ahmed), OP.CIT. P450. N²2542.

4 - GÓMEZ-JORDANA (Sonia), Analyse syntaxique et sémantique de quelques proverbes français et espagnols du XIVe au XXIe siècle, In Bulletin Hispanique, V 111, $\mathrm{n}^{\circ} 2$ 2, 2009, P590. On dit aussi (Quand on veut noyer son chien, on dit qu'il a la rage). 


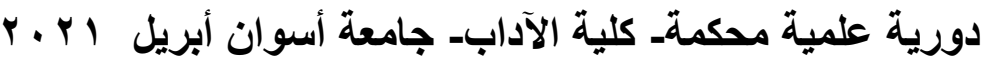

"On trouve toujours un prétexte quand

on est décidé à se débarrasser de

quelqu'un ou de quelque chose ${ }^{(1)}$.

Plusieurs facettes du proverbe qu'on trouve dans la culture française. Lorsqu' on est en train de parler d'une personne qui arrive soudainement, on dit :

\begin{tabular}{|c|c|c|}
\hline $\begin{array}{l}\text { Proverbe } \\
\text { français }\end{array}$ & Equivalent arabe & Translittération \\
\hline $\begin{array}{c}\text { «Quand on } \\
\text { parle du loup, on } \\
\text { en voit la } \\
\text { queue » }\end{array}$ & | ـ "جبنا سيرة القط جه ينط")("إفتكرنا القط جه ينط & 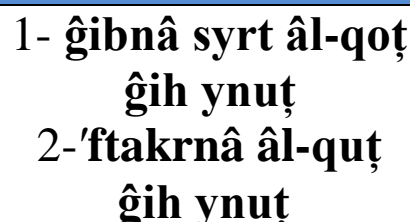 \\
\hline
\end{tabular}

Dans les deux cultures (française et arabe), le loup et le chat, les deux animaux qui symbolisent la vitesse, occupent une place essentielle dans l'histoire du proverbe. Le loup s'utilise dans plusieurs proverbes pour montrer des messages variés. En exprimant le courage de l'homme qui fait face aux problèmes, on trouve :

Quelquefois, on dit :

«Tenir le loup par les oreilles » ${ }^{(4}$

«Prendre le taureau par les cornes ${ }^{(5)}$

En effet, le proverbe représente tous les domaines de la vie quotidienne. Quand la nécessité oblige souvent 1'homme à faire des choses contre son habitude, on dit ce proverbe :

\begin{tabular}{|c|c|c|}
\hline $\begin{array}{c}\text { « La faim chasse le } \\
\text { loup hors du bois » }\end{array}$ & Al-ĝguc kâfir \\
\hline
\end{tabular}

\section{Autres formes du même contexte}

1 - AL ATTAR (Abdallah), OP.CIT. P 231.

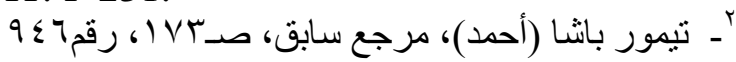

- TAYMOR BACHA (Ahmed), OP.CIT. P173. N946.

3 - QUITARD (P.M), OP.CIT. P605.

4 -_ABDELHADI (Mohammed), OP.CIT. P168, N855.

5 - DE BOUCHE À OREILLE, Recueil d'expressions idiomatiques, Volume

3, Réalisé par un groupe d'élèves en formation de base commune présecondaire sous la supervision de Sylvia Impala, Paris, Larose. 2010. P15.

6 __ABDEL HADI (Mohammed), OP.CIT. P 94, N 488 


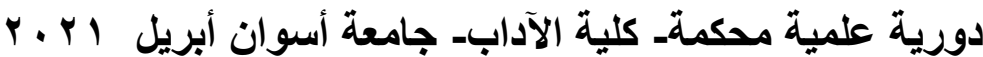

\begin{tabular}{|c|c|c|}
\hline $\begin{array}{c}\text { 1-« Nécessité n'a pas de } \\
\text { loi »(1). } \\
\text { 2-«Ventre affamé n'a } \\
\text { point d'oreilles » } \\
\text { 3- « Besoin ne garde } \\
\text { loi » }{ }^{(3)} \text {. }\end{array}$ & 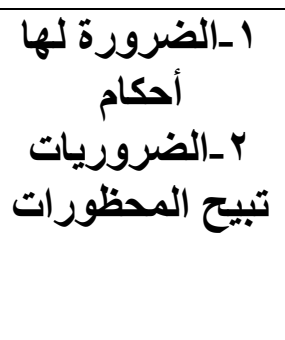 & $\begin{array}{c}\text { 1-Al-ḍarurah lahâ } \\
\text { 'ḥkâm } \\
\text { 2-Al-ḍaruryât tubyh } \\
\text { âl-maḥzurât }\end{array}$ \\
\hline
\end{tabular}

Verbe «chasser » exprime la sortie obligatoire du loup qui vit loin des hommes, mais quand il avait faim, il n'hésite pas à quitter le bois pour manger, c'est-à-dire, celui qui est dans le besoin fait n'importe quoi (en période de guerre, les règles ne sont pas respectées). A cet égard, nous pouvons citer une petite histoire : un jour, Napoléon a visité une école. En sortant, Il disait aux élèves :

«Jeunes gens, souvenez-

vous bien que chaque heure

du temps perdu est une

chance de malheur pour

l'avenir. Ce sont des mots

remarquables d'un homme

qui connaissait toute la

valeur du temps ${ }^{(4)}$.

Ces mots certifient que le passé nous donne toujours l'expérience qui aide à continuer. Pour immortaliser cette idée, les français disent :

\begin{tabular}{|c|c|c|}
\hline Proverbe français & Equivalent arabe & Translittération \\
\hline $\begin{array}{l}\text { «e temps pe } \\
\text { se rattrape jan }\end{array}$ & فات مـات" & 'Ili \\
\hline
\end{tabular}

Parmi les proverbes de la culture arabe, nous trouvons aussi :

1 - QUITARD (P.M), OP.CIT. P340

2 -LA FONTAINE (Jean),_Fables choisies mises en vers, quatrième partie, Fable «Le Milan et le Rossignol », Claude Barbin et Denys Thierry, Paris, $1679, \mathrm{P} 3$.

3 - ABDEL HADI (Mohammed), OP.CIT. P20, N 10.

4 - QUITARD (P.M), OP.CIT. P363.

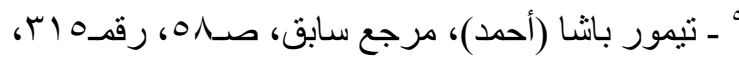

- TAYMOR BACHA (Ahmed), OP.CIT. P320. N¹766.

6 - ABDEL HADI (Mohammed), OP.CIT. P116, n 611. 


\section{اللى راح مبيرجش \\ ('lli râh mabyrg š)}

Alors, Il est impossible d'arrêter le temps ou de faire retourner le passé. Le conseil est donc d'oublier, de ne pas se reprocher, de ne pas regretter car «tout regret à son sujet est inutile » ${ }^{(1)}$. Ces expériences nous accordent cette sagesse :

$$
\text { «Avec l'âge on devient sage } »^{(2}
$$

En arabe, on trouve l'équivalent qui dit :

$$
\text { "اللى ما تربيه الأهالى تربيه الأيام و الليالي" }
$$

('lli mâ trabyh âl-'hâly trabyh âl-'yâm w âl-lyâly)

Ce proverbe nous conseille : il faut obéir à ceux qui nous présentent des conseils (surtout les parents) pour que l'homme puisse affronter les obstacles de la vie dont la joie et le chagrin représentent les deux ailes.

Aujourd'hui, nous sommes heureux, demain, il est probable que nous serons tristes. La tristesse n'est pas éternelle, comme le bonheur ne vient pas chaque jour. Cette idée semble évidente dans

\begin{tabular}{|c|c|c|c|}
\hline $\begin{array}{l}\text { "Les jours se suivent et } \\
\text { ne se ressemblent pas }{ }^{(4}\end{array}$ & "الدنيا بدل يوم بصل & & $\begin{array}{l}\text { unyâ badal } \\
\text { csal w yum } \\
\text { bașal. }\end{array}$ \\
\hline \multicolumn{4}{|c|}{ Autres formes du même contexte } \\
\hline $\begin{array}{l}\text { 1- Ce n'est pas tous les } \\
\text { jours fêtes } \\
2-\ll \text { Tel qui rit vendredi, } \\
\text { dimanche pleurera }{ }^{(1}\end{array}$ & إك ويوم عليك & & $\begin{array}{l}\text { Yum lak w } \\
\text { yum clyk. }\end{array}$ \\
\hline
\end{tabular}
le proverbe suivant :

1 -_NATAF (Gilda), CHAMBARD (Roger), GRAILLE (Barbara), BOUCHERIT (Aziza), Proverbes libyens, Gellas Karthala, Paris ,2002, P12, N47.

2 - ABDELLAH ADI (Mohamed). OP.CIT. P16, N89.

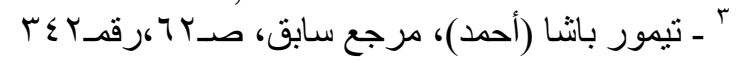

- TAYMOR BACHA (Ahmed), OP.CIT. P62. N³42.

4 - QUITARD (P.M), OP.CIT. P483

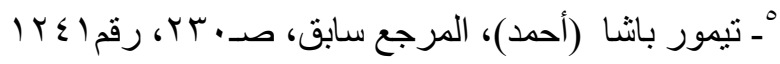

- TAYMOR BACHA (Ahmed), OP.CIT. P230. N 1241. 


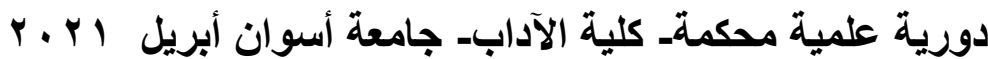

L'homme doit être prudent parce que la vie n'est pas toujours belle. Il faut présenter l'aide pour tous parce qu'on ne sait demain on aura besoin de qui :

«Gratte-moi l'épaule et je t'en ferai autant » ${ }^{(2)}$

En général, l'argent ne fait pas le bonheur. Il y a sûrement le plus important (la santé, les enfants, le travail, etc....). Quand on est en bonne santé, on peut travailler pour gagner l'argent. La santé est donc le vrai trésor :

«La santé n'est pas l'absence de la maladie,

c'est un sentiment plus profond que le bien-

être qui ne dépend pas seulement des services

de santé, mais du travail, du revenu, de

l'éducation, de la culture des droits et des

libertés »(3).

Cette idée se montre claire dans le proverbe suivant :

\begin{tabular}{|c|c|c|}
\hline $\begin{array}{l}\text { « Beaucoup de } \\
\text { richesses, } \\
\text { beaucoup de } \\
\text { soucis » }\end{array}$ & كل ما تكثر القلوس تكتر & $\begin{array}{l}\text { Kul mâ tktar âl-flus } \\
\text { tktar humumhâ }\end{array}$ \\
\hline \multicolumn{3}{|c|}{ Autres formes du même contexte } \\
\hline $\begin{array}{l}\text { «Suffisance fait } \\
\text { richesse » }(5)\end{array}$ & 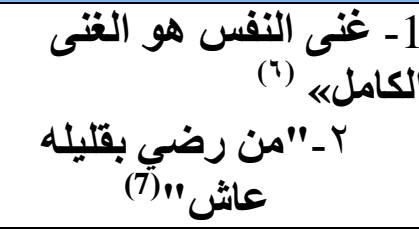 & $\begin{array}{l}\text { 1- Ģnâ âl-nafs hua } \\
\text { âl-ġnâ âl-kâml } \\
\text { 2- Min rộ bi- } \\
\text { qalyluh câš }\end{array}$ \\
\hline
\end{tabular}

${ }^{1}$-ODDO (Alexandra), Syntaxe des proverbes binaires: coordinations et parataxes Syntax of Binary Proverbs: Coordinations and Parataxes, In Rilce. Revista de Filología Hispánica, V34, N², 2018, P485.

2 - AL ATTAR (Abdallah), OP.CIT. P76.

3 - M. (Mrabet), Définitions, approches et concepts en santé publique, Santé publique-Master USSAC,2018. P7.

4- ABDEL HADI (Mohammed), OP.CIT. P20, N 107.

5 - Ibid. P103.

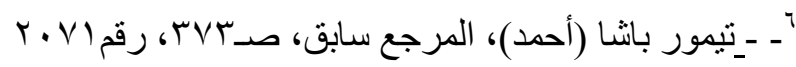

- TAYMOR BACHA (Ahmed), OP.CIT. P373. N²071.

-Ibid. P496. $\mathrm{N}^{\circ} 2820$.

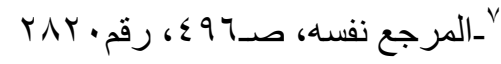


La question du travail se répète plus d'une fois dans l'univers des proverbes. Outre l'importance du travail, une outre facette apparait: travailler est essentiel pour gagner l'argent et pour réaliser le progrès pour toute l'humanité. Le proverbe suivant décrit ironiquement l'état des ouvriers qui ne se profitent pas de leurs métiers comme le dentiste qui a mal aux dents, la couturière qui habille des anciens vêtements :

\begin{tabular}{|c|c|c|}
\hline $\begin{array}{l}\text { «Les cordonniers } \\
\text { sont les plus mal } \\
\text { chaussés » }\end{array}$ & الاسكافى حافي & Al-'skâfy ḥâfy \\
\hline \multicolumn{3}{|c|}{ Autres formes du même contexte } \\
\hline أناس ولعى عريانة"(") & ז-"زى الإبرة تك اب & $\begin{array}{l}\text { 1- Bâb âl-nĝâr mihlac } \\
\text { 2- Zay âl-'brah tiksy âl- } \\
\text { nâs w hy cryânah }\end{array}$ \\
\hline
\end{tabular}

Dans l'équivalent, le mot «nue » symbolise la couturière qui malgré la fabrication des vêtements pour tous, porte un costume déchiré.

Aux instants de colère, l'homme ne peut pas penser ou bien il pense négativement. En proverbe, on dit :

\begin{tabular}{|c|c|c|}
\hline Proverbe français & $\begin{array}{c}\text { Equivalent } \\
\text { arabe }\end{array}$ & Translitération \\
\hline «Prendre la mouche ${ }^{(5)}$ & "زعناخهيره" طأن & $\begin{array}{l}\text { z'luh 'lâ țarf } \\
\text { mnâhyyruh }\end{array}$ \\
\hline
\end{tabular}

Le mot «mouche» désigne «un souci». En outre, le verbe «prendre » signifie «prendre ombrage »:

1 - AL ATTAR (Abdallah), OP.CIT. P 40.

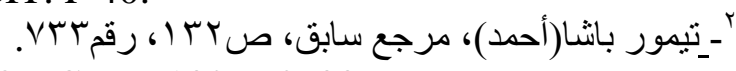

- TAYMOR BACHA (Ahmed), OP.CIT. P132. Nº733.

- Ibid. P251. $\mathrm{N}^{\circ} 1361$

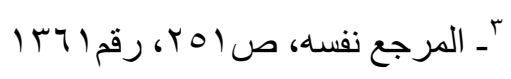

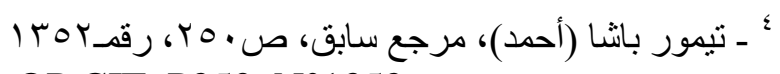

- TAYMOR BACHA (Ahmed), OP.CIT. P250. N 1352

5 - QUITARD (P.M), OP.CIT. P543. 


\section{دورية علمية محكمة كلية الآداب- جامعة أسوان أبريل ا ب r.}

«La mouche désigne un souci, une

pensée négative qui arrivait

brusquement. Dans cette expression.

Le verbe "prendre" a le sens de

"prendre ombrage" »"

Ce proverbe s'adresse à celui qui se vexe pour une raison futile. D'ailleurs, on utilise le mot «mouche» pour signaler «un petit insecte volant ${ }^{(2)}$ :

\section{Conclusion :}

$$
\text { «Entendre une mouche voler }{ }^{(3}
$$

$\mathrm{Au}$ terme de ce chapitre, on peut dire que la traduction se définit conformément à " Ses apports très pertinents d'internationalisation de connaissance, d'interpénétration des cultures et civilisations et de popularisation de sciences et technologies ${ }^{(4)}$. La traduction n'est pas l'explication de mots mais elle est considérée comme un moyen pour trouver la même idée du texte dans la langue arrivée :

«En fait, la traduction ne peut pas se réduire au passage d'une langue à une autre : elle nécessite toujours une adaptation complète $d u$ document d'origine à un public qui se caractérise par des habitudes différentes, des goûts différents, des modes de pensée différents, des comportements différents. Un public, donc, qui devra recevoir le document traduit comme si ce dernier

1 - https://www.linternaute.fr/expression/langue-francaise/344/prendre-lamouche. Consulté le 22-1-2020.

2 - REY-DEBOVE (Josette), REY (Alain), OP.CIT. P1643.

3 - https://www.linternaute.fr/expression/langue-francaise/19838/entendreune-mouche-voler. Consulté le 22-1-2020.

4. JOSEPH WALIYA (Yohanna), OP.CIT. P1. 


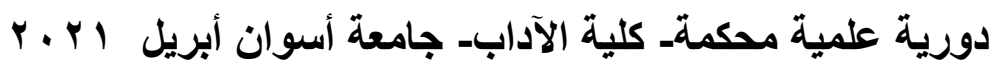

avait été rédigé par quelqu'un de même culture. " ${ }^{(1}$

En bref, nous pouvons dire que la traduction n'est qu'une évasion du sens lexicographique en donnant l'idée générale du texte. Par ailleurs, nous remarquons également que la lexicographie nous aide à arriver au sens global ${ }^{(2)}$. En ce qui concerne les problèmes de la traduction, c'est le manque de compétences pour discriminer entre les expressions qui sont soit traduisibles soit intraduisibles ainsi que techniques et spécialisés. En outre, nous nous confondrons aussi aux théories et aux procédés de la traduction.

D'ailleurs, ce chapitre nous a permis de découvrir plusieurs aspects et caractéristiques parémiologiques du proverbe arabe et français. Les équivalents précédents affirment que les proverbes sont généralement le point de rencontre de plusieurs expériences issues à une morale d'une mémoire vivante. Alors, nous concluons qu'il y a un échange interculturel parémiologique entre les deux cultures arabe et française, aboutissant l'apparition d'équivalences trouvées pour la plupart des proverbes cités cihaut. En outre, La transmission du discours proverbial exige essentiellement une traduction basée sur l'équivalence et l'adaptation.

1 -GOUADEC(Daniel), Le traducteur, la traduction et l'entreprise. Afnor, Paris1989. p.16.

2 - la lexicographie nous aide à chercher les emplois des mots pour arriver les sens visés. 


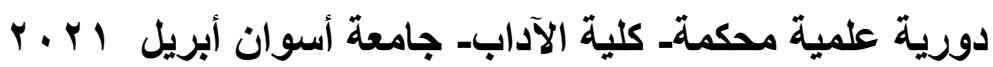

\section{Bibliographie \\ I-Corpus}

-ABDEL HADI (Mohammed), « Recueil de proverbes francais et leurs équivalents en arabe et en anglais », Egypte, IMP. Salah El Dine Alex 1933.

- QUITARD (Pierre Marie), « Dictionnaire étymologique, Historique et Anecdotique, des proverbes et des locutions proverbiales de la langue francaise $»$, Paris, P. Bertrand libraireéditeur, 1842.

\section{II-Ouvrages consacrés aux proverbes}

- MOY (Sylvie), « 100 Proverbes francais », Franc-Parler, Sénas, France, 2012.

- NILLA (Sowmiya), «Les proverbes à travers le monde ", ÉLODIL, paris 2006.

-QUITOUT (Michel) \& MUNOZ (Julia Sevilla), « Traductologie, proverbes et figements », L'Harmattan, paris, 2009.

\section{III-Ouvrages consacrés à la traduction}

- GOUADEC(Daniel), « Le traducteur, la traduction et l'entreprise », Paris, Afnor1989.

\section{IV-Articles}

-CENDRIER (Patrick), «Des chiens et des hommes », In Publibook, France,2003.

-CHACOTO (LUCÍLIA MARÍA), «Figement et variation dans les proverbes portugais », In Paremia, V6, Madrid, 1997. -CONDE TARRÍO (Germán), «La littérature et le proverbe, le proverbe et la littérature ", Universidade de Santiago de Compostela, In Littérature, langages et arts: rencontres et création, 2017.

- HERMAN (Jan), «L'écrivain au 18e siècle : Voltaire dans son époque », In : in Ecrivains Modes d'emploi ; De Voltaire à bleu Orange, Musée royal de Mariemont, Sofiane Laghouati, Morlanwelz, 2012. 


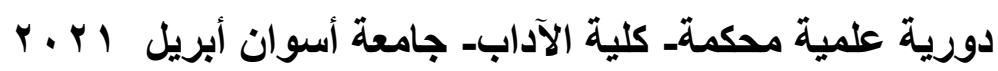

- INMACUADA (María), «Enseigner et apprendre les unités parémiologiques d'une langue étrangère : du XIXe siècle à nos jours », In Cédille, $\mathrm{N}^{\circ} 10$, Université de Rovirai Virgili, Espagne 2014.

-MOHAMAD El SAYED El TOUKHY (Dalia), «L'interculturalité entre le proverbe populaire égyptien et francais », In Magazine de Faculté des lettres de linguistiques et de cultures comparées, V12, N², Egypte, 2020.

- PRIVAT (Maryse), «A propos de la traduction des proverbes », In Revista de Fitología Romaníca , número 15 , janvier 1998.

-SEVILLA MUÑOZ (Julia), «Les proverbes et les phrases proverbiales francaises et leurs équivalents en espagnol », In : Langages, 34 année, $\mathrm{n}^{\circ} 139,2000$.

\section{V-Romans}

-Molière, «le médecin malgré lui », théâtre du palais -Royal, paris 1666 , Acte $1, \mathrm{Sc} 2$.

- BALZAC (Honoré de), «Eugénie Grandet », Alexandre Houssiaux, Éditeur, paris, 1855.

VI-Thèses consultées

- SAAD ALI (Mohamed), «En attendant Godot de Samuel Beckett, traduction en arabe et étude littéraire et linguistique, Faculté des Lettres », Thèse de Magistère, Université de Zagazig, Egypte, 2008.

\section{VII- conférence}

-René Klein (jean), « la phraséologie dans le trésor de la langue française informatisé. Conférence présentée le 24 mai 2006 dans le cadre du «Séminaire de méthodologie en étymologie et histoire du lexique », France, Nancy, 2007.

\section{VIII-Dictionnaires}

- BERTAUD DU CHAZAUD (Henri), « Dictionnaire de synonymes et contraires », les usuels, paris, 2001. 
- DUBOIS (Jean), et alii, « Dictionnaire de linguistique »,

LAROUSSE, paris, 1994.

- MALOUX (Maurice), «Dictionnaire des proverbes, sentences,

et maximes », paris VI ${ }^{\mathrm{e}}$, Librairie Larousse, 1950.

-MONTREYNAUD (Florence), PIERRON (Agnès), FRANCOIS (Suzzoni), «Dictionnaire de proverbes et dictions, la sagesse du monde entier », paris, Poche, 2015.

- MORVAN (Danièle), REY (Alain), « $\underline{\text { Dictionnaire } \boldsymbol{L E}}$

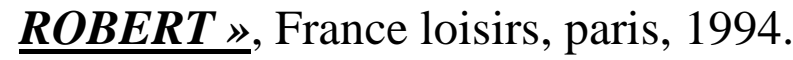

\section{IX-Sitographies}

-http://www.linternaute. -http://www.cultivonsnous.fr.

-https://coran12-21.org/fr/sourates/s23.

-https://books.openedition.org/psorbonne/17529.

= https://www.expressions-francaises.fr/expressions

$$
\begin{aligned}
& \text { مصادر بالعربية } \\
& \text { ـأمين عبد الصمد (محمد)، القيم فحى الامثال الشعبية بين مصر وليبيا، القاهرة، }
\end{aligned}
$$

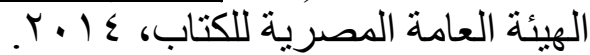

$$
\begin{aligned}
& \text { تيمور باشنا (أحمد)،أومثال العامية، الطبعة الثانبة، مصر، دار الكتاب العربي، } \\
& 1907 .
\end{aligned}
$$

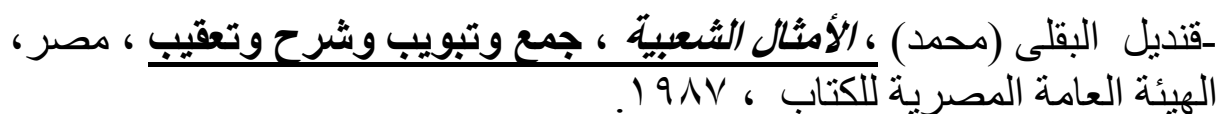

$$
\begin{aligned}
& \text { قو قاميس } \\
& \text { ـ العطار (عبداله)، الأمثال الشعبية الفرنسية، مصر ، أطلس للنشر و الإنتاج }
\end{aligned}
$$

ـعبد النور (جبور)، إدريس (سهيل)، قاموس المنهل فرنسي عربي، الطبعة الثامنة،

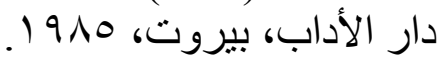

